\title{
Content Based Image Retrieval based on Domain Knowledge Acquisition
}

\author{
Feroza D Mirajkar, Ruksar Fatima, Shaik A Qadeer
}

\begin{abstract}
Enormous growth has been noted in digital technology over the years and this has led to massive growth in data mainly images and videos, hence with the volume of the data has also increased. Moreover, searching and retrieval of the accurate image from the huge dataset has become eminent as well as one of the challenging issue. In order to solve mainly two technique were used i.e. based on text and content. Moreover, content based is technique that is more efficient. In past several technique were used but they lack from the efficiency. Hence, in this paper we have proposed a methodology based named as DKA (Domain Knowledge Acquisition), DKA focuses on two main goals first is learning the similarities across the varied domain and other is learning on complex objects. The main advantage of DKA is the whether the object is whether from same domain or the other the retrieval process is very smooth. Moreover In order to evaluate the performance we have retrieved the top 5 response of query image and later it is compared with the several state-of-art technique and the existing method based on the precision parameter.
\end{abstract}

Keyword: CBIR, DKA, Image retrieval

\section{INTRODUCTION}

Due to extensive connectivity and easily availability of digital imaging technologies and internet, multimedia files have increased exponentially and data sharing, searching and posting has become very flexible. Hence, these multimedia data is used for real time video as well as audio conferencing. Moreover, this has led the creation of massive image from the various fields, at the same time the need for the image retrieval tool has become one of the fundamental requirement.

There are two types of image retrieval is used namely text based retrieval and content based image retrieval [1]. Moreover, in the early day's text based approach was used, however this method leads us to the increase in size and it results in being subjective [2]. Hence in the early 90s wide research has resulted in development of CBIR i.e. Content based Image retrieval [3], here the image retrieval were Based on the AM (Automated Matching) through the similarity evaluation. CBIR (Content Based Image Retrieval) is the bottleneck when it comes to accessing the multimedia databases; the main intention behind the CBIR is to retrieve the similar image $[4,5]$. There exist two major problem which is quiet difficult to solve the issue, first is the segmentation of images i.e. when the image is parted into several meaningful parts based on the LL-(Low Level) feature such as spatial, shape ,texture and color.

Revised Manuscript Received on November 08, 2019.

Prof. Feroza D Mirajkar, Assistant professor, Department of Electronics and Communication Engineering, Khaja Banda nawaz college of engineering, Kalaburagi.

Dr. Ruksar Fatima, HOD computer science engineering, vice-principal, Khaja Banda Nawaz College of engineering, Kalaburagi.

Dr. Shaik A Qadeer, Professor, Muffakham Jah College of Engineering and Technology, Hyderabad.
Second issue is the marginal gap between the high-level image and low-level image in case of the bus, home, furniture and others. Hence, it is required to design an efficient method for large image datasets.

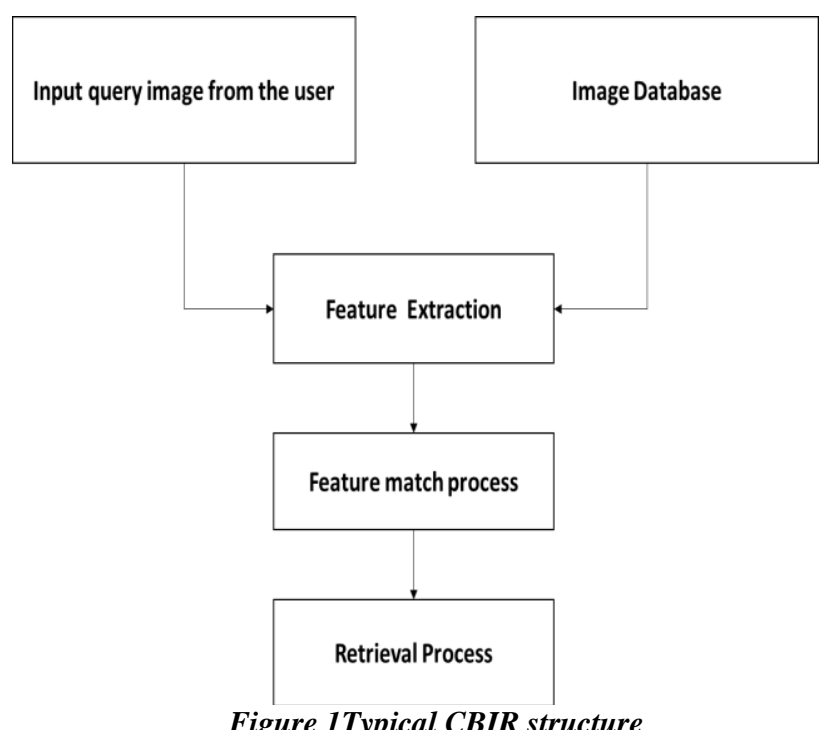

Figure 1Typical CBIR structure

The above figure shows the typical architecture of CBIR model, where there are two primary stages that are involved namely Feature extraction and feature matching.[6] Moreover feature extraction involves in extracting the feature and in feature matching, features are compared to match. Moreover, the image feature of each image is stored in the available image dataset. If it is found that the distance between the images available in the database and FV (Feature Vector) of particular query is small then it is said that the query image matches from the image datasets. Later these matched images are ranked according to the similarity index. Moreover, in past several algorithm has been proposed and it focuses on the iteration-based scheme however, they have failed miserably to answer few scenarios such as how many rounds of iterations are required or about the manifold structure. Hence, to unfold that we have proposed a model, which is known as DKA (Domain Knowledge Acquisition) which is regularization, based model. Moreover, with the help of regularization DKA process obtains the similarity index. Moreover, to make the computation more feasible we have used iteration. Moreover, later we have used the context where the size of the domain no longer matters. 


\section{Content Based Image Retrieval based on Domain Knowledge Acquisition}

\section{LITERATURE SURVEY}

[7] presented a corresponding high-level query language, PICQUERY, [8] described an approach where local statistics (texture energy measures) are estimated at the output of an equivalent filter bank by means of a nonlinear transformation (absolute value) followed by an iterative Gaussian smoothing algorithm. An integrating system of faces and fingerprints was developed by developed by Anil Jain and Lin Hong [9] which overcomes the limitations of both face and fingerprint recognition systems. [10] Attempted to capture high-level concepts from low-level image features under the constraint that the test image does belong to one of the classes.[11] presented their work in the area of image organization and retrieval in Image Databases using global color features and spatial color distribution of images. They suggested extending the use of image histograms to characterize the global and local color properties of an image, moreover here the artificial neural network were used.

[12] Proposed a method for concept-based medical image retrieval that is a superset of existing semantic-based image retrieval methods. They conceive of a concept as an incremental and interactive formalization of the user's conception of an object in an image. [13] Propose a hybrid image descriptor that is rotation and scale-invariant to make image retrieval efficient. The quantization based on the RGB color space is used to extract the color features, whereas the patterns generated from locally structuring element are assembled to extract the texture features. The color and texture features are integrated together to build the hybrid feature descriptor known as rotation and scaleinvariant hybrid descriptor (RSHD). The RSHD descriptor is robust in case of rotation and scaling. [14] Proposes an innovative image descriptor to extract the color and texture features of the image. This descriptor is known as global correlation descriptor (GCD). The color feature is characterized using global correlation vector (GCV), whereas the texture feature is characterized using directional global correlation vector (DGCV). Experimental results show that the performance of the GCD outperforms as compared with the recent CBIR techniques. [15] Propose a framework that uses affine image moment invariants as a feature descriptor for image retrieval. The moments are fed into the BoW model to produce feature vectors. The novelty of this work is that the affine moment invariants are used as local features and are extracted using SURF feature descriptor. Similarly in [16] meta-heuristic approach were used such as iterative local search and genetic algorithm and the methodology was evaluated with investigation of number of images.

Zeng et al. [17] propose an image representation technique in which Gaussian mixture models (GMMs) are employed to characterize an image as a spectrogram (i.e. general histogram of colors). The expectation-maximization (EM) technique is employed to determine quantized color space from the training data set. Gaussian components, that is, the number of color bins, are characterized according to the
Bayesian information criterion (BIC). Spatiogram is modified and incorporated into a quantized Gaussian mixture color model. Finally, a comparative analysis of two spectrograms is done and a new measurement adopting technique is suggested known as Jensen-Shannon divergence (JSD) to make image retrieval more efficient. Zhao et al. [18] propose a descriptor based on the local and multi-trend structure for feature representation called multitrend structure descriptor (MTSD). This descriptor integrates features like color, edge orientation, and shape and intensity information for robust image representation. It also represents the local spatial structure information to extract image features. The experimental results demonstrated that MTSD produced discriminative results for effective CBIR. a hybrid method was presented by integrating local and global features The local features are extracted by applying SIFT descriptor, while the global features are extracted by applying upper-lower of LBP (ULLBP) descriptor based on LBP. Then, features are quantized into the BoW model to improve image retrieval performance. Although LBP is not suitable for color images, it is not suitable for capturing similarity between color images. LBP only captures textual information from the images. Liu et al. [19] overcame this problem by proposing a descriptor with an additional color feature called color information feature (CIF) incorporated with LBP for image retrieval. Experimental results show that combining these two features yields good performance in a retrieval system. [20] Propose a CBIR method that combines LBP and wavelet transform. In this method, the texture features of the image are extracted by computing LBP codes using the coefficients of discrete wavelet transform (DWT). Then, shape features are extracted from texture features by computing Legendre moments using these LBP codes to construct a feature vector. The experimental results show improved performance on small image databases as compared with the large image databases. Bala and Kaur [21] propose a novel descriptor for CBIR called local texton XOR patterns (LTxXORP). Then, the LTxXORP feature vector is integrated with the feature vector of the HSV colour space-based colour histogram to improve CBIR performance. A novel method using a low-level feature called composite moment (CoMo) for image retrieval is proposed by Vassou et al. [22]. This method combines moment invariants and colour unit of the colour and edge directivity descriptor (CEDD). This method improves CBIR performance by overcoming rotation, translation and scaling issues. 
III.

\section{PROPOSED METHODOLOGY}

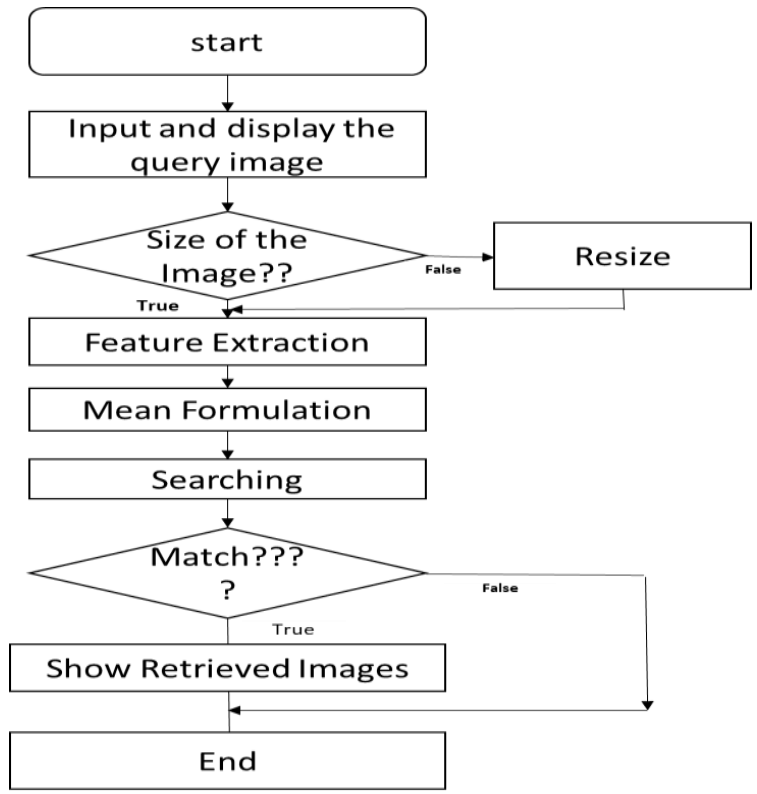

Figure 2 Proposed flow-work.

The above diagram i.e. fig 2 shows the flow of our process, at first user inputs the query in the form of image, this image is displayed. The displayed image is checked for the size , if the size is compatible then it goes to the $\mathrm{FE}$ (Feature Extraction) phase, in here the initial feature of the image is used as CFV(Compact feature Vector). Once the feature extraction is done mean is formulated, this in terms helps in searching the image. If the image is matched then show the desired images else displayed as error

\section{Shape Feature}

Let there be any two datasets of $2 \mathrm{D}$ shape $D=D_{z}, Z=$ $1, Y \subset M^{2}$ and $\mathrm{A}=a_{i}, i=1, \ldots \ldots, B \subset M^{2}$, here the main intention is to choose the best spatial deformation among others i.e. $s^{*}: M^{2} \rightarrow M^{2}$ along with that the best correspondence is also chosen between the two given two dataset.

$$
\begin{gathered}
(C, d \mathrm{f})=\sum_{k=1}^{B} \sum_{z=1}^{Y} c_{z, k}\left\|A_{k}-s\left(d_{z}\right)\right\|^{2}+\lambda\|D S\|^{2} \\
+\operatorname{Temp} \sum_{k=1}^{B} \sum_{z=1}^{K} c_{z, k} \log c_{z, k} \\
-\varsigma \sum_{k=1}^{B} \sum_{z=1}^{Y} c_{z, k}
\end{gathered}
$$

Here $C=\left(c_{z, k}\right)_{Y}$ is Responsibility Accountability Matrix, this matrix describes the occurrence of correspondence between the given datasets, this in terms satisfies the following two scenarios i.e.

$$
\sum_{k=1}^{B+1} c_{z, k}=1
$$

And

$$
\sum_{z=1}^{y+1} c_{z, k}=1
$$

For all $c_{z, k}$ which belongs to the boundary of $[0,1]$. Later, in order to handle the various outliers extra rows and columns are added. In equation $1, \mathrm{D}$ be the laplacian operator and $\mathrm{s}$ be the SD (spatial deformation). Similarly, in order to assure the deformation smoothness the regularity term is used and it is denoted by using the $\|D S\|^{2}$. Temperature is dentoted by degree of correspondence is controlled by the parameter temperature and denoted by Temp. Moreover for balancing the accuracy along with the smoothness is denoted by the parameter $\lambda, \varsigma$ is used for restricting the outliers.

$\|D S\|^{2}=\iint\left[\left(\frac{\partial^{2} s}{\partial \chi^{2}}\right)+2\left(\frac{\partial^{2} s}{\partial \chi \partial y}\right)+\left(\frac{\partial^{2} s}{\partial y^{2}}\right)\right] d \chi d y$

Hence, our algorithm can be written through updating the correspondence, which is done by calculating $c_{z, k}$

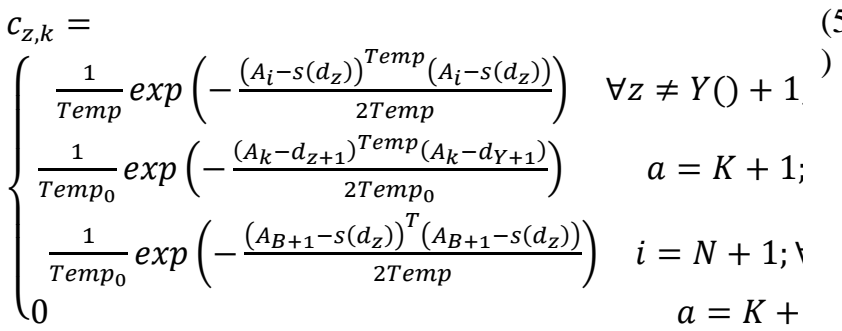

Here, $v_{K+1}$ and $\chi_{N+1}$ are said to be the cluster center of the given outliers, then the norms of $\mathrm{M}$ through rows and column is given by below equation. Once the rows and column are update we tend to update the transformation, the reduction in energy is given by the below equation.

$$
\begin{gathered}
c_{z, k}=\frac{c_{z, k}}{\sum_{l=1}^{Y+1} c_{l, k}}, \quad k=1,2, \ldots \ldots \ldots, B, \\
c_{z, k}=\frac{c_{z, k}}{\sum_{o=1}^{B+1} m_{a, j}}, \quad k=1,2, \ldots \ldots \ldots, Y, \\
E_{T P S}(f)=\sum_{k=1}^{B} \sum_{z=1}^{Y} \begin{array}{c}
c_{z, k}\left\|\begin{array}{c}
A_{k} \\
-S\left(d_{z}\right) \\
+\lambda\|D S\|^{2},
\end{array}\right\|^{2}
\end{array}
\end{gathered}
$$

$$
S\left(d_{z}, e, F\right)=\phi\left(d_{z}\right) \times \omega+d_{z} \times e
$$

In the above Energy equation, there exist two matrices, which is decomposed by deformation $S$. In here, $\mathrm{d}$ is said to be the affine matrix and $\omega$ is non-affine matrix, this claeraly indicates the updation of $S$ deformation by updating the $S$ and.$s$ Deformation is decomposed into two parts i.e. $s_{1}$ and $s_{2}$, the relation between $\mathrm{A}$ and $\mathrm{g}$ can be represented by $s=s_{1} \oplus s_{2}$. Here, $s_{1}$ and $s_{2}$ are the nonlinear and linear transformation respectively, between the given data sets. Similarly the mean of these two transformation is given as $s_{1}\left(d_{z}\right)=s_{1} d_{z}$ and $s_{2}\left(s_{1}\left(s_{2_{1}}\left(d_{z}\right)\right)\right)=\left(s_{2_{1}}\left(s_{1}()\right)\right)$

$$
\begin{aligned}
G\left(C, s_{1}, s_{2}\right)=\sum_{k=1}^{B} & \sum_{z=1}^{Y} c_{z, k}\left\|A_{k}-s_{1}\left(d_{z}\right)-s_{2}\left(d_{z}\right)\right\|^{2} \\
& +\lambda\left\|D s_{2}\right\|^{2} \\
& +\operatorname{Temp} \sum_{i=1}^{N} \sum_{a=1}^{K} c_{z, k} \log c_{z, k} \\
& -\varsigma \sum_{i=1}^{N} \sum_{a=1}^{K} c_{z, k}
\end{aligned}
$$




\section{Content Based Image Retrieval based on Domain Knowledge Acquisition}

From the above equation, it is clear that the local nonlinear deformation $\mathrm{g}$ and affine transformation $\mathrm{A}$ can be easily decoupled and when it is reduced, the reduction can be formed in tow sub-problems, which can be represented in the two sub-problems.

$$
\begin{array}{rl}
s_{1}{ }^{y+1}=\arg \min _{s_{1}} & G\left(C^{y}, s_{1}, s_{2}{ }^{y}\right) \\
= & \sum_{k=1}^{B} \sum_{z=1}^{Y} c^{z}{ }_{z, k} \| A_{k}-s_{1}{ }^{y}\left(s_{1}\left(d_{z}\right)\right) \\
- & s_{1}\left(d_{z}\right) \|^{2} \\
\left(C^{y+1}, s_{2}{ }^{y+1}\right)= & \arg \min G\left(C^{y}, s_{1}{ }^{y+1}, s_{2}\right) \\
& =\sum_{k=1}^{B} \sum_{z=1}^{Y} c_{z, k} \|{\widetilde{A_{k}}}^{y+1} \\
& -s_{2}\left(s_{1}{ }^{y+1} s_{1}\left(d_{z}\right)\right) \|^{2} \\
& +\lambda\left\|D s_{2}\right\|^{2} \\
& +T e m p \sum_{k=1}^{B} \sum_{z=1}^{Y} c_{z, k} \log c_{z, k} \\
& -\varsigma \sum_{k=1}^{B} \sum_{z=1}^{Y} c_{z, k}
\end{array}
$$

\section{SIFT}

SIFT is one of the feature detection algorithm for detecting as well as describing the local features in the given images. In a given feature point, the descriptor of SIFT formulates and calculates the GV (Gradient Vector) and later it creates the normalized histogram of the GD (Gradient Directions). This is achieved by using the partition process, i.e. SIFT Descriptor is divided into the particular resolutions of the given pixels. In case of each pixel, gradient vector of that particular pixel is added to the gradient directions. This is achieved through the process of quantization. Furthermore, the gradient direction is weighed using the Gaussian scale, later these weighted values are shared to the neighboring bins this helps in minimizing the BE (Boundary effects) between the orientation and positions.

\section{DKA (Domain Knowledge Acquisition) Process}

Domain knowledge acquisition can be used for retrieving the images; the main aim of algorithm is to get the novel sim(similarity matching). Similarity matching is the term, which is used, for how much both the objects are similar, comparison is done and the image retrieved are ranked based. Let us consider any weighted graph $W G=(G, H)$ that models the data manifold here graph vertices represents the data points $G=\left\{g_{1}, g_{2}, \ldots, g_{M}\right\}$ and $H \in A^{M \times M}$ is the matrix of adjacent graph. $H_{t u}$ is the $\operatorname{sim}$ between the $h_{l}$ and $h_{m}$ defined as given in the below equations. Moreover, our intention is to identify the novel similarity, which is denoted byNSim. Initially model learns the similarity on the direct product graph. In DPG (Direct Product Graph), DPG each edge describes the four vertices whereas each of the vertex shows the relations between the given two vertices. In this research work the direct product graph is used and it is denoted by $(G X H) \cdot D P G=(K, L)$ Can be defined as $K=G X G, L=H \otimes H$.Moreover the main aim is to learn the novel similarity measure i.e. $\operatorname{sim}=\{\operatorname{sim}\}_{1 \leq l, m \leq M}$.

\section{Problem Definition}

The problem definition of the particular research work is presented through the below equation, here the intention is to present the novel similarity index measurement.

$$
\begin{gathered}
\min _{\text {NSim }} 0.5 \sum_{a, b, c, d=1}^{M} H_{b c} H_{c d}\left(\frac{N \operatorname{Sim}_{c a}}{\left(D M_{a a} D M_{c c}\right)^{\frac{1}{2}}}\right. \\
\left.-\frac{N \operatorname{Sim}_{d b}}{\left(D M_{b b} D M_{d d}\right)^{\frac{1}{2}}}\right)^{2} \\
+\mu \sum_{c a=1}^{B}\left(N \operatorname{Sim}_{c a}\right. \\
\left.-D V_{c a}\right)^{2}
\end{gathered}
$$

In order to achieve the novel similarity below optimization can be used and the below equation represents it .Here $\mu$ is regularized parameter, along with its elements $D M_{g g}=$ $\sum_{u=1}^{B} H_{a b}$ diagonal matrix is represented by using $D M$. Whereas domain values are denoted by using the $D V$ which belongs to $Z^{M \times M}$. Equation presents problem definition, moreover our methodology i.e. DKA process mainly contain two process, in first process influence of matching $\mathrm{P}$ on the given matching sim is described. Moreover, this phenomenon is called $h$ proc. Smooth proc Is described as if the $g_{k}$ matches to $x_{l}$ then probability of them falling in same category should have the negligible difference and the similarities $M_{t t}$ and $M_{t t}$ remains siimlar. Moreover, the individual object. GRK (Graph based Ranking) model helps in data ranking with res Second term is known as the fitting term shown in equation 1 this gives the difference from the initial. Moreover achieving the finite solution for the equation is very difficult hence two distinctive operators are used

namely

Vectorization $(V)$ and inverse vectorization(IV). Two product of Cartesian coordinate model i.e. $\gamma$ and $\delta$ where these two can be computed by the below equation.

$$
\alpha=\mathrm{M}(\mathrm{e}-1)+\mathrm{j}
$$

$\delta=\mathrm{M}(\mathrm{g}-1)+\mathrm{l}$ 
Moreover the smooth proc is written as

$$
\begin{aligned}
& \frac{1}{2} \sum_{\alpha, \delta=1}^{M^{2}} H_{\alpha \beta}\left(\frac{\overrightarrow{N s ı m}_{\alpha}}{\sqrt{D M_{\alpha \alpha}}}-\frac{\overrightarrow{N s ı m}_{\delta}}{D M_{\delta \delta}}\right)^{2} \\
& =\sum_{\alpha, \delta=1}^{M^{2}} L_{\alpha \delta} \frac{\overrightarrow{N S l m}_{\alpha}^{2}}{D M_{\alpha \alpha}} \\
& -\sum_{\alpha, \delta=1}^{N^{2}} \overrightarrow{N S \iota m}_{\alpha} \frac{L_{\alpha \beta}}{\left(D M_{\alpha \alpha} D M_{\delta \delta}\right)^{1 / 2}} \overrightarrow{N S \iota m}_{\delta} \\
& =\sum_{\alpha=1}^{M^{2}} \overrightarrow{N S \imath m}_{\alpha}^{2}-\overrightarrow{N s ı m}^{\mathbb{T}} D M^{-1 / 2} L D M^{-1 / 2} \vec{V} \\
& =\overrightarrow{N S \imath m}^{T}\left(U M-D M^{-1 / 2} D M^{1 / 2}\right) \vec{V} \\
& =\overrightarrow{N S \imath m}^{T}(U M-B) \overrightarrow{N S \imath m},
\end{aligned}
$$

Where UM is the unit matrix.

$$
\begin{gathered}
L=G \otimes G \in Z^{M^{2} X M^{2}} \\
D M D=D M \otimes D M \in Z^{M^{2} X M^{2}} \\
\mathrm{~B}=\mathrm{E} \otimes \mathrm{E} \in Z^{M^{2} X M^{2}}
\end{gathered}
$$

$\mathrm{B}=D M D^{-1 / 2} D M D^{-1 / 2}$

Moreover, there are three sub-stages are applied when the transformation takes place, first is if $\mathrm{W}$ is symmetric then $\mathbb{W}$ can also be symmetric, second process is depicted through below equations

Moreover the objective functions are given through the below equation

$$
\text { of }=\overrightarrow{N S \imath m}^{T}(u m-B) \overrightarrow{N S \imath m}+\mu\|\overrightarrow{N S \imath m}-\vec{F}\|^{2}
$$

Partial derivative of the above equation i.e. eq.5 is taken with respect to $\overrightarrow{N S \imath m}$, which is shown in the below equation (eq6), $u m-B$ is the normalized direct product graph

$$
\frac{\partial o f}{\partial \overrightarrow{N S \imath m}}=2(u m-B) \overrightarrow{N S \imath m}+2 \mu(\overrightarrow{N S \imath m}-\vec{F})
$$

The above equation is considered as zero then we get below equation. Later we applying the vectorization to both side of equation we get the equation $18 \cdot \vec{V}$ is a function that has a real value and provides to the each vetex which describes the relationship. Henceforth DKA takes admittance matrix as the smooth operator that helps in securing the structure. Moreover, our intention is to utilize the direct product graph

$$
\overrightarrow{N s ı m}=\frac{\mu}{\mu+1}\left(u m-\frac{1}{\mu+1} B\right)^{-1} \vec{F} .
$$

$$
\begin{aligned}
\operatorname{NSim}^{*}=(1-\alpha) & 1 \\
& / \operatorname{vector}(1 \\
& /(T-\alpha E \otimes E) \operatorname{vector}(B)),
\end{aligned}
$$

$$
=(1-\alpha) 1 / \text { vector }(1 /(I-\alpha B) \vec{F})
$$

NSim $^{*}$ is the feasible solution,

\section{DKA Iteration based}

Since the computation of DKA using the certain number is complex, hence we apply the iteration-based approach, in order to perform the iteration we initialize the $A^{(1)}$. In here the similarity values

Limit

$$
\begin{gathered}
\sum_{a=1}^{\infty}(\alpha E)^{i}=(u m-\alpha B)^{i} \\
B^{*}=\sum_{i=1}^{\infty}(\alpha B)^{i}
\end{gathered}
$$

$B^{*} \in Z^{M^{2} X M^{2}}$ Moreover our intention is to learn a novel similarity index $\boldsymbol{N S i m}^{*} \in \boldsymbol{Z}^{\boldsymbol{M}^{2} \boldsymbol{X} \boldsymbol{M}^{\mathbf{2}}}$ hence it is required to collect he few portion and place it to the NSim* and this can be achieved through below equation.

$$
N \operatorname{Sim}^{*}=\operatorname{vec}^{-1}(B * \vec{F})
$$

Here $Y$ depicts the indices of particular elements in $\boldsymbol{B}^{*}$

\section{Measuring based interpretation}

$$
\begin{aligned}
\mathrm{B}_{\mathrm{i}}=\left[\mathrm{E}_{\mathrm{i} 1}, \mathrm{E}_{\mathrm{i} 2}, \ldots, \mathrm{E}_{\mathrm{iN}}\right] & \in \mathrm{Z}^{1 \mathrm{XM}} \\
& <\mathrm{E}_{\mathrm{c}}, \mathrm{E}_{\mathrm{d}}>=\sum_{\mathrm{a}, \mathrm{b}=1}^{\mathrm{M}} \mathrm{NSim}_{a b}^{(\mathrm{t})} B_{c a} B_{d a}
\end{aligned}
$$

Moreover $\mathrm{E}_{\mathrm{c}}$ and $\mathrm{E}_{\mathrm{d}}$ norms are omitted.

\section{Variants}

\section{Learning on heterogeneous graph}

Two data domains are considered namely $W G_{1}$ and $W G_{2}$ and given as $W G_{1}=\left(G_{1}, H_{1}\right)$ and $\left(G_{2}, H_{1}\right)$ where $G_{i}$ is the data-points in the given particular domain, similarity is derived through $N$ Sim $\in Z^{Z^{M_{1} X M_{2}}}$, it measures the matching of objects between two different domain. Let $g_{c}$ and $g_{d}$ be the two objects in the first domain and the $g_{a}$ and $g_{b}$ be the two objects in another domain. Moreover, the objective is given as:

$$
\begin{aligned}
\min 1 / 2 \sum_{\mathrm{a}, \mathrm{b}=1}^{M_{2}} \sum_{\mathrm{c}, \mathrm{d}=1}^{M_{1}} H_{a b}^{2} \mathrm{H}_{\mathrm{cd}}\left(\frac{\mathrm{NSim}_{\mathrm{ca}}}{\left(D M_{a b}^{2} D M_{c c}^{1}\right)^{1 / 2}}\right. \\
\left.-\frac{\mathrm{NSim}_{\mathrm{db}}}{\left(D M_{b b}^{2} D M_{d d}^{1}\right)^{1 / 2}}\right) \\
+\mu \sum_{\mathrm{i}=1}^{M_{2}} \sum_{\mathrm{k}=1}^{M_{1}}\left(\mathrm{NSim}_{\mathrm{ca}}\right. \\
\left.-\mathrm{F}_{\mathrm{ca}}\right)^{2}
\end{aligned}
$$




\section{Content Based Image Retrieval based on Domain Knowledge Acquisition}

Here $\mu$ is the RP (Regularization Parameter) and $\mathrm{Y}$ is the CM (Cross-Model) similarity, moreover by applying $g$ the co-ordinate transformation the above equation can be written as

$$
\operatorname{NSim}^{(t+1)}=\alpha \operatorname{SNSim}^{(t)} E^{(t)}+(1-\alpha) F
$$

Moreover the above scenario is suitable only for the inside domain retrieval, Moreover cross model domain is retrieved though the below process.

\section{Learning on Direct product Graph}

Let $W G=(G, H)$ be the hypergraph with $E d$ hyperactive edges and $\mathrm{M}$ vertices, the graph of the vertices are denoted with the $\mathrm{M}$ hyper edges and $\mathrm{M}$ vertices. Moreover the graph vertices denotes the given data points $\mathrm{G}=\left\{g_{1}, g_{2}, g_{3}, \ldots \ldots, g_{M}\right\}$. In here every Hyper edges $\epsilon$ in $W G$ holds some weight and these weights are stored in the matrix $H \in Z^{M X E d}$ these graphs are denoted through the incidence matrix i.e. IMATEZ $Z^{N X M}$ as

$$
\operatorname{IMAT}(\mathrm{a}, \epsilon)=\left\{\begin{array}{lr}
1, & \text { if } g_{a} \in \epsilon \\
0 & \text { if } g_{a} \in \epsilon
\end{array}\right.
$$

Vertex Degree is given in below figure

$$
\mathrm{DMD}_{\mathrm{ii}}=\sum_{\epsilon=1}^{\mathrm{Ed}} \mathrm{G}_{\epsilon \epsilon} \mathrm{IMAT}_{\mathrm{i} \epsilon}
$$

Moreover Edge degree is given as in the below equation and EDEG

$$
\mathrm{EDEG}_{\epsilon \epsilon}=\sum_{\mathrm{i}=1}^{\mathrm{M}} \mathrm{IMAT}_{\mathrm{i} \epsilon}
$$

Let $\mathrm{DMD} \in Z^{M X M}$ be the similarity matrix hence the objective is to optimize the process.

$\min 1$

$/ 2 \sum_{\epsilon \in=1}^{\mathrm{Ed}} \sum_{\mathrm{a}, \mathrm{b}, \mathrm{c}, \mathrm{d}=1}^{\mathrm{M}} \frac{\mathrm{H}_{\epsilon \epsilon} \mathrm{IMAT}_{\epsilon \epsilon} I M A T_{\mathrm{a} \epsilon} I M A T_{\mathrm{b} \epsilon} \mathrm{IMAT}_{\epsilon \epsilon} \mathrm{IMAT}}{\mathrm{EDEG}_{\epsilon \epsilon} \mathrm{EDEG}_{\epsilon \epsilon}}$

Moreover, in order to solve the above equation three coordinate transform that are identical which are presented in the below three equation.

$$
\begin{gathered}
\alpha=M(a-1)+c \\
\beta=M(j-1)+l \\
\gamma=E d(\epsilon-1)+\varepsilon
\end{gathered}
$$

Moreover in the above equation the transformation utilization takes place by substituting though following

$$
\begin{gathered}
H_{\epsilon \epsilon} H_{\epsilon \epsilon}=L_{\gamma \gamma} \\
I M A T_{a \epsilon} I M A T_{c \epsilon}=I M A T_{\alpha \gamma} \\
I M A T_{b \epsilon} I M A T_{d \epsilon}=I M A T_{\beta \gamma} \\
E D E G_{\epsilon \epsilon} E D E G_{\epsilon}=W_{\gamma \gamma} \\
B_{\gamma \gamma}=\sum_{\beta=1}^{M^{2}} I M A T_{\beta \gamma}
\end{gathered}
$$

$$
D M D_{\alpha \alpha}=\sum_{\gamma=1}^{E d^{2}} L_{\gamma \gamma} I M A T_{\alpha \gamma}
$$

Moreover the above equation helps in achieving the smooth procedure and given through the below equation

$$
\begin{aligned}
& \overrightarrow{N s ı m}(u m \\
& \left.-D M D^{-\frac{1}{2}}(I M A T) L(E D E G)^{T} D M D^{-\frac{1}{2}}\right) \overrightarrow{N s ı m}
\end{aligned}
$$

\section{PERFORMANCE EVALUATION}

DKA method are coded in MATLAB on the i7 processor packed with 2 GB Nvidia Graphics and 8 GB RAM through windows10 to evaluate the algorithm. The evaluation is done in wo part first part depicts the Query and response of image retrieval another part is comparison based on the precision

\section{Datasets}

In this research work we have used the Corel1k Datasets, $\mathrm{CD} 1 \mathrm{~K}$ is the integration of ten different natural scene these are: African, Bus, Beach, Food, Flowers, Dinosaurs, Elephants, Horses , Buildings and Mountains. These images have the same size of $384 \mathrm{X} 256$ and each image categories consists of 100 images, hence the CD1K contains the total number of images are 1000 .

\section{Comparative analysis}

In order to evaluate the DKA process we have used the precision parameter, precision which is as positive predictive value is defined as relevant instances fraction in retrieved instance. moreover in other words we can say that precision is relevance measure. Precision is calculated by using the below formula.

$$
\text { Precision }=\frac{\text { relevent } \text { image retrieved }}{\text { Total } \text { images retrieved }}
$$

Below table 1 shows the comparative analysis of various existing methods, moreover the comparison is done on the 10 different natural scene and average value of precision is tabulated in the last raw of table 1. Moreover, our methodology i.e. DKA is compared against the several stateof-art technique namely MTH [23], MSD [24], CDH [25], SED [26], GCD [27], MCMCM [28] and the existing methodology CPV-THF [29]. The average precision value of each methodology is calculated and tabulated in the last raw. We observe that precision value of MTH is $68.25 \%$, MSD is $72.167, \mathrm{CDH}$ is $65.75 \%$, SED is $61.58 \%, \mathrm{GCD}$ is $72.5 \%$ and the existing system i.e. CPV-THF is $80.79 \%$ whereas proposed system has the precision value of $94.611 \%$. This indicates that our method has marginal improvisation in terms of precision value. 
Table 1: comparison of various methods with proposed methodology

\begin{tabular}{|l|l|l|l|l|l|l|l|l|}
\hline \multirow{2}{*}{ Image_Category } & \multicolumn{9}{|c|}{ Precision value (in \%) } \\
\cline { 2 - 10 } & $\begin{array}{c}\text { MTH } \\
{[\mathbf{2 3}]}\end{array}$ & $\begin{array}{c}\text { MSD } \\
{[\mathbf{2 4}]}\end{array}$ & $\begin{array}{c}\text { CDH } \\
{[\mathbf{2 5}]}\end{array}$ & $\begin{array}{c}\text { SED } \\
{[\mathbf{2 6}]}\end{array}$ & $\begin{array}{c}\text { GCD } \\
{[\mathbf{2 7}]}\end{array}$ & $\begin{array}{c}\text { MCMCM } \\
{[28]}\end{array}$ & $\begin{array}{c}\text { CPV- } \\
\text { THF[29] }\end{array}$ & $\begin{array}{l}\text { roposed } \\
\text { Model }\end{array}$ \\
\hline African & 69.17 & 83.33 & 77.5 & 82.5 & 87.5 & 69.75 & 91.66 & 93.1 \\
\hline Beach & 61.67 & 43.33 & 56.67 & 28.33 & 68.33 & 54.25 & 54.58 & 93.1 \\
\hline Building & 45.83 & 63.33 & 47.5 & 47.5 & 61.67 & 63.95 & 78.75 & 98.08 \\
\hline Bus & 68.33 & 76.67 & 71.67 & 73.33 & 80 & 89.65 & 86.25 & 91.67 \\
\hline Dinosaur & 100 & 100 & 100 & 90 & 100 & 100 & 98.7 & 98.08 \\
\hline Elephant & 70.83 & 65 & 62.5 & 55 & 67.5 & 48.8 & 65.83 & 96.3 \\
\hline Flower & 75 & 86.67 & 60.83 & 72.5 & 88.33 & 92.3 & 95.41 & 100 \\
\hline Horse & 100 & 97.5 & 91.67 & 62.5 & 100 & 89.45 & 93.33 & 100 \\
\hline Mountain & 39.17 & 29.17 & 44.17 & 40 & 55 & 47.3 & 56.25 & 87.98 \\
\hline Food & 52.5 & 76.67 & 45 & 64.17 & 74.17 & 70.9 & 85.83 & 87.88 \\
\hline Average & 68.25 & 72.167 & 65.75 & 61.58 & 72.85 & 72.5 & 80.79 & 94.61 \\
\hline
\end{tabular}

\section{Performance evaluation on Corel1K dataset}

In order to prove the robustness of DKA methodology is proved through retrieving the image, moreover we have used the Corel1 $\mathrm{K}$ dataset, which consist of 10 different nature scene each containing 100 images. Moreover, here we have presented the top 5 response of the query, In the below figure we see that in case of African category the Figure 3 represents the query image of African category and figure $4 a, 4 b, 4 c, 4 d$ and $4 \mathrm{e}$ are the response of input query. Similarly, Figure 5 represents the query image of beach and Figure $6 a, 6 b, 6 c, 6 d$ and $6 e$ are the response image of beach. Figure 7 is the query image of building and Figure $8 \mathrm{a}, 8 \mathrm{~b}, 8 \mathrm{c}, 8 \mathrm{~d}$ and $8 \mathrm{e}$ are response of the image.

\section{Category: African}

Query image: 1
Figure 9 is query image of Bus and Figure 10a, 10b, 10c, $10 \mathrm{~d}$ and $10 \mathrm{e}$ are the response of the image. Figure 11 is the query image of dinosaur and figure $12 \mathrm{a}, 12 \mathrm{~b}, 12 \mathrm{c}, 12 \mathrm{~d}$ and $12 \mathrm{e}$ are the response of the image. Figure 13 is the query image of elephant and the figure 14a, 14b, 14c, 14d and 14e are response image. Figure 15 is the query image of flower and Figure 16a, 16b, 16c, 16d and 16e are the image response of flower. Figure 17 is the query image of horse and the $18 \mathrm{a}, 18 \mathrm{~b}, 18 \mathrm{c}, 18 \mathrm{~d}$ and $18 \mathrm{e}$ are the image response. Figure 19 is the query image of mountain and figure 20a, 20b, 20c, 20d and 20e are the image response of same, Figure 21 is the query image of food and the top response of the image response is figured in $22 \mathrm{a}, 22 \mathrm{~b}, 22 \mathrm{c}, 22 \mathrm{~d}$ and $22 \mathrm{e}$.

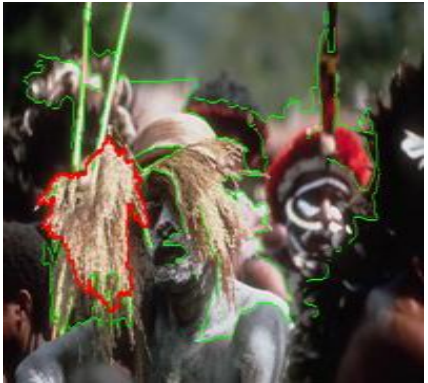

Figure 3 query image

Image Response: 


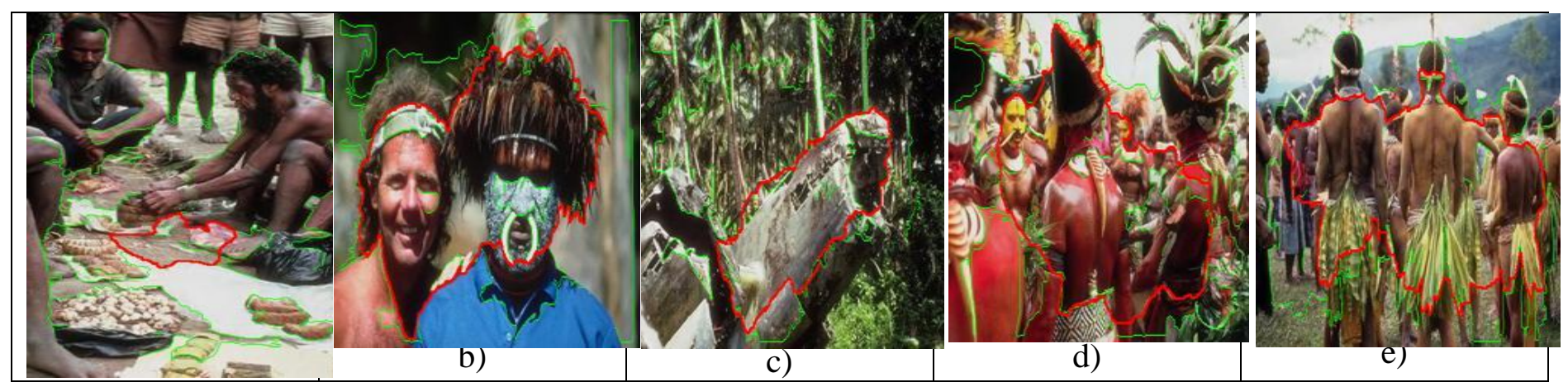

Figure 4 Response Image from datasets

\section{Quary image:2}



\section{Category: Beach}

Figure 5: Quary image

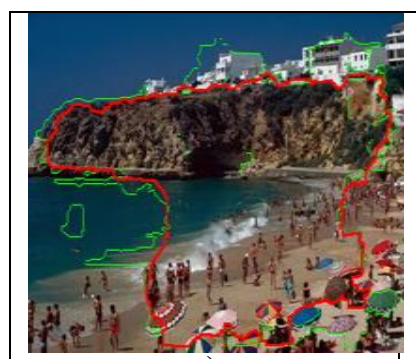

a)



b)

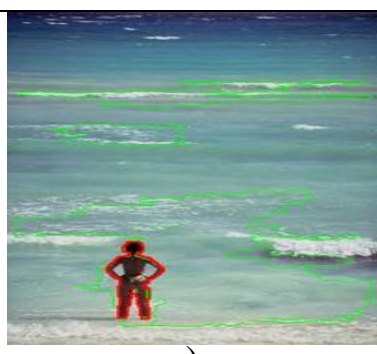

c)

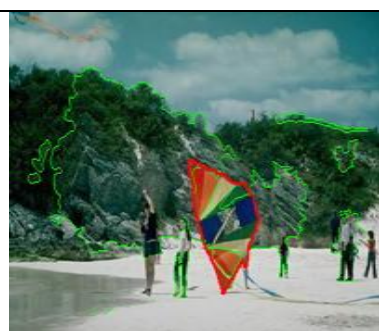

d)

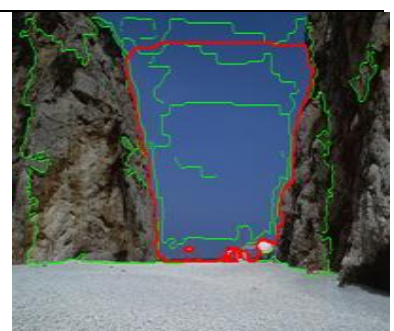

e)

Figure 6 Top 5 response of beach images

\section{Category: Building}

Query image:3

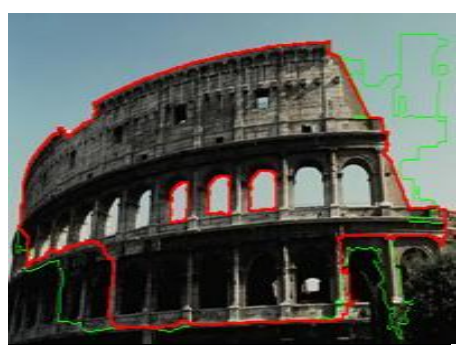

Figure 7 Query image of building Images

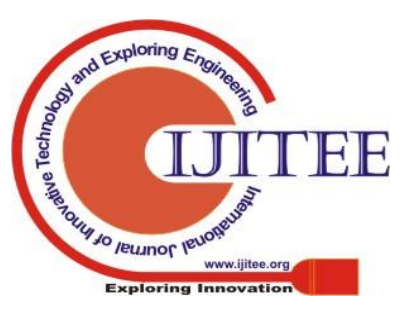




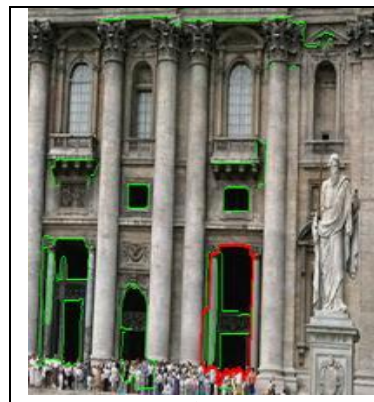

a)

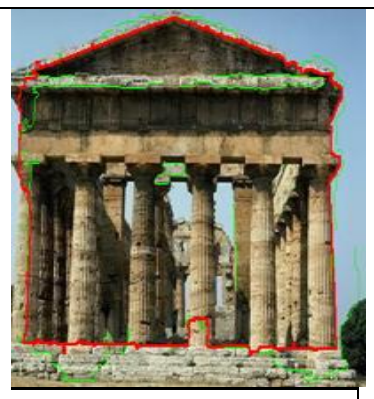

b)

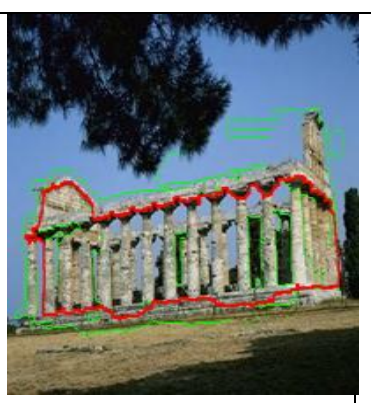

c)

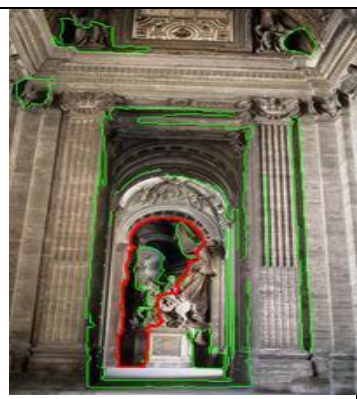

d)

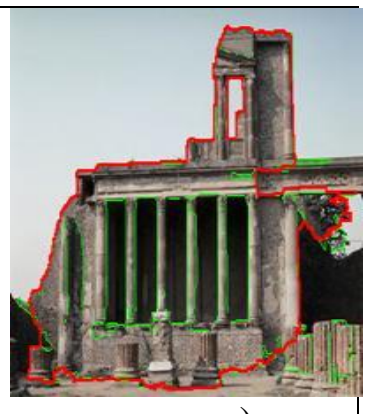

e)

Figure 8 Top 5 response image of building category

Query image 4:

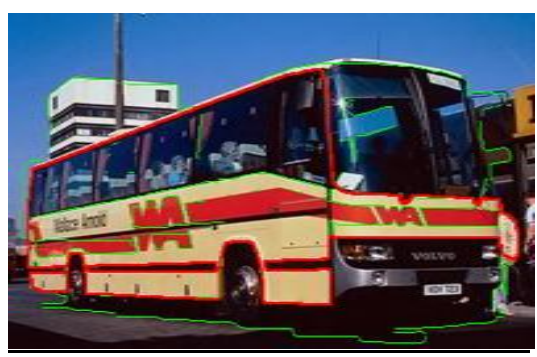

Figure 9 query image of bus category



a) b)

Figure 10 Top 5 response image of Bus Category

c)

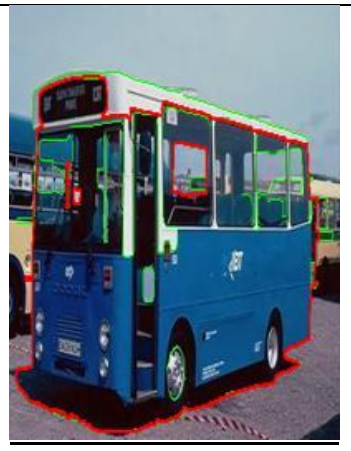

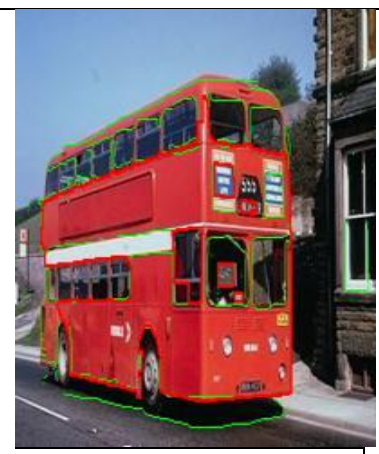

d)



e)

Query image 5

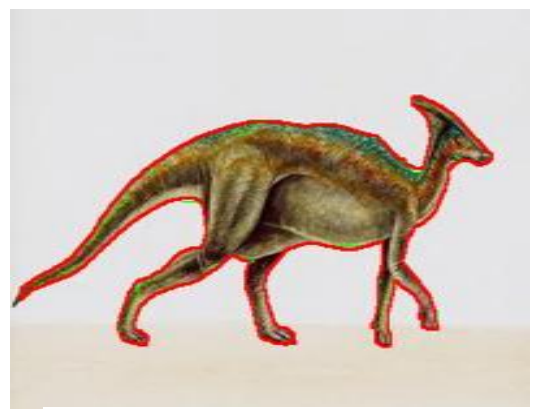

Figure 11 Query Images for dinosaur






\section{Content Based Image Retrieval based on Domain Knowledge Acquisition}



Figure 12 top 5 response images of dinosaur

Query image 6

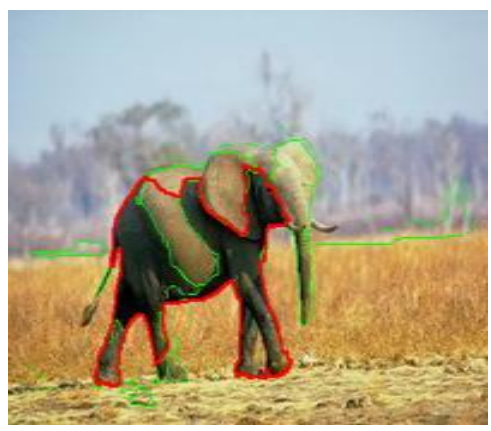

Figure 13query image for elephant category



a)

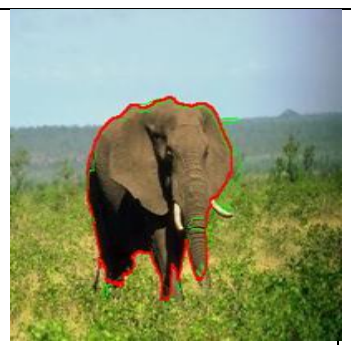

b)
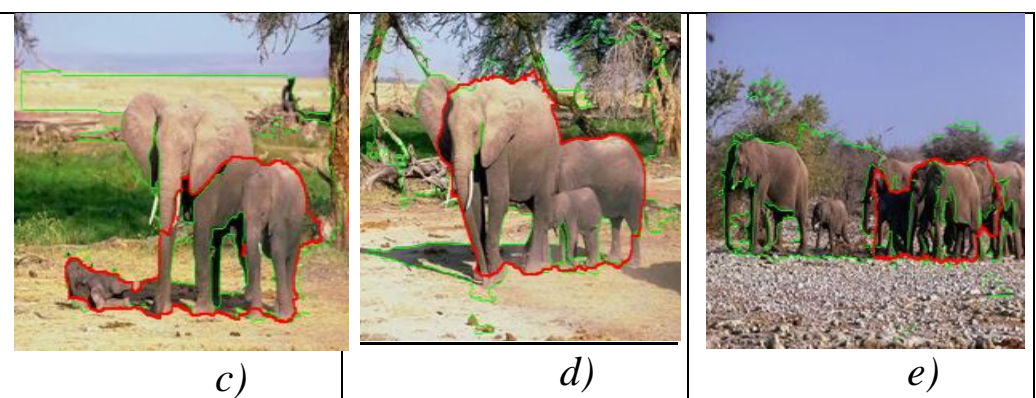

Figure 14 Response images of Elephants category

Query image 7

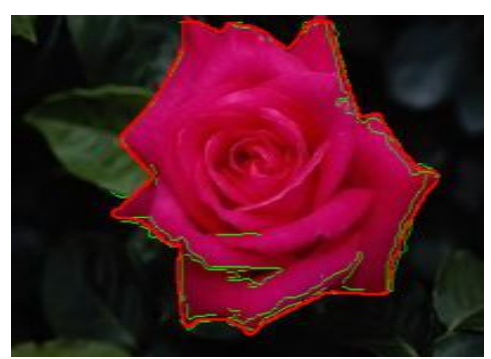

Figure 15 Query image of flower 


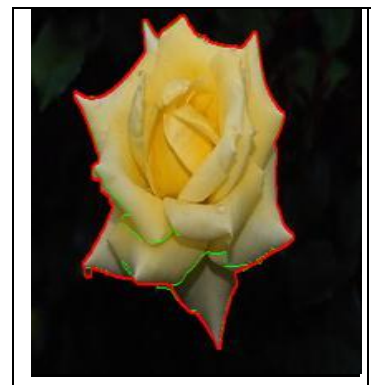

a)

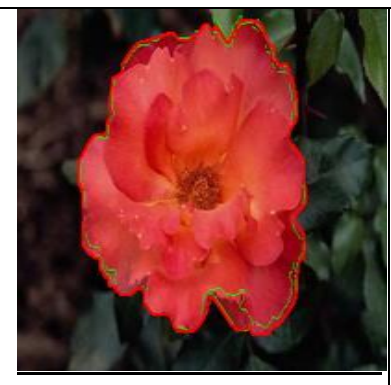

b)

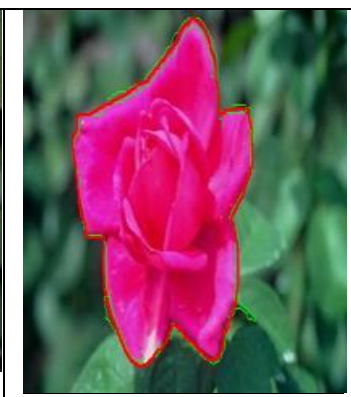

c)

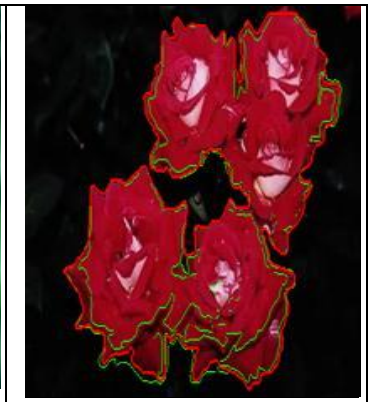

d)

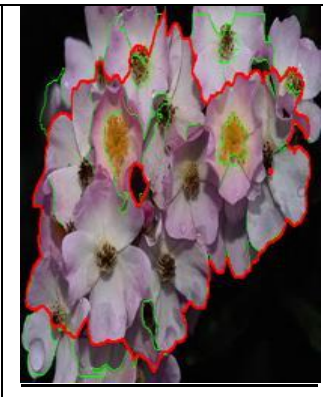

e)

Figure 16 Response image of Flower

Query image 8

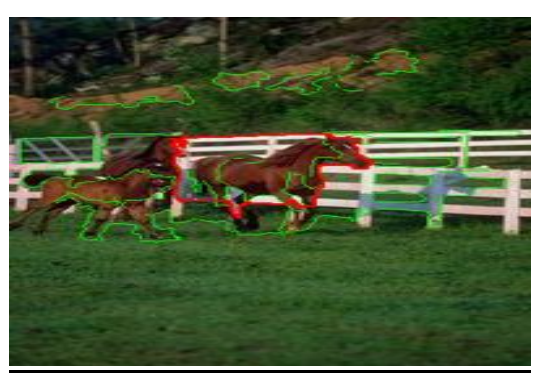

Figure 17Query Image of Horse

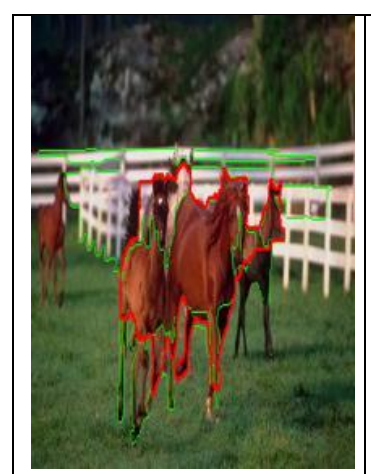

(a)

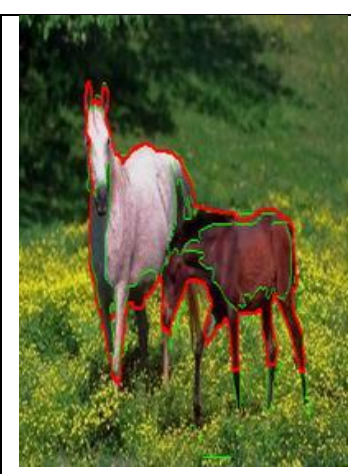

(b)

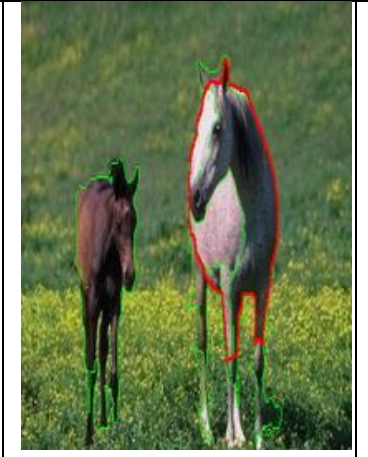

$\underline{(\mathrm{c})}$

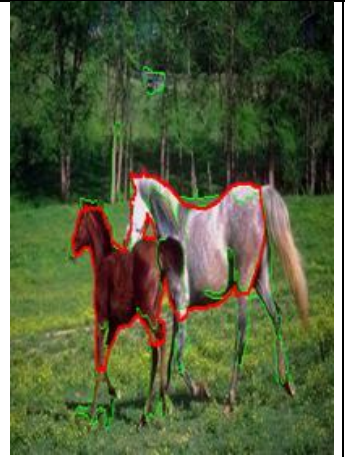

(d)

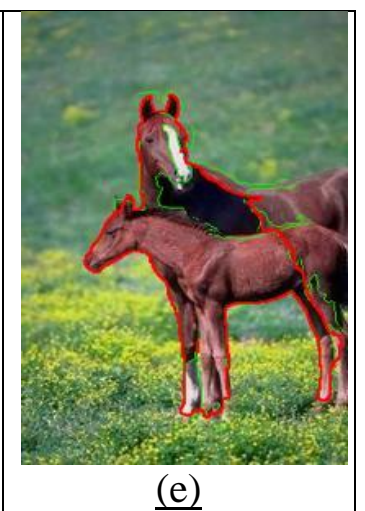

(e)

Figure 18 Response Image of Horse Category

Query Image 9
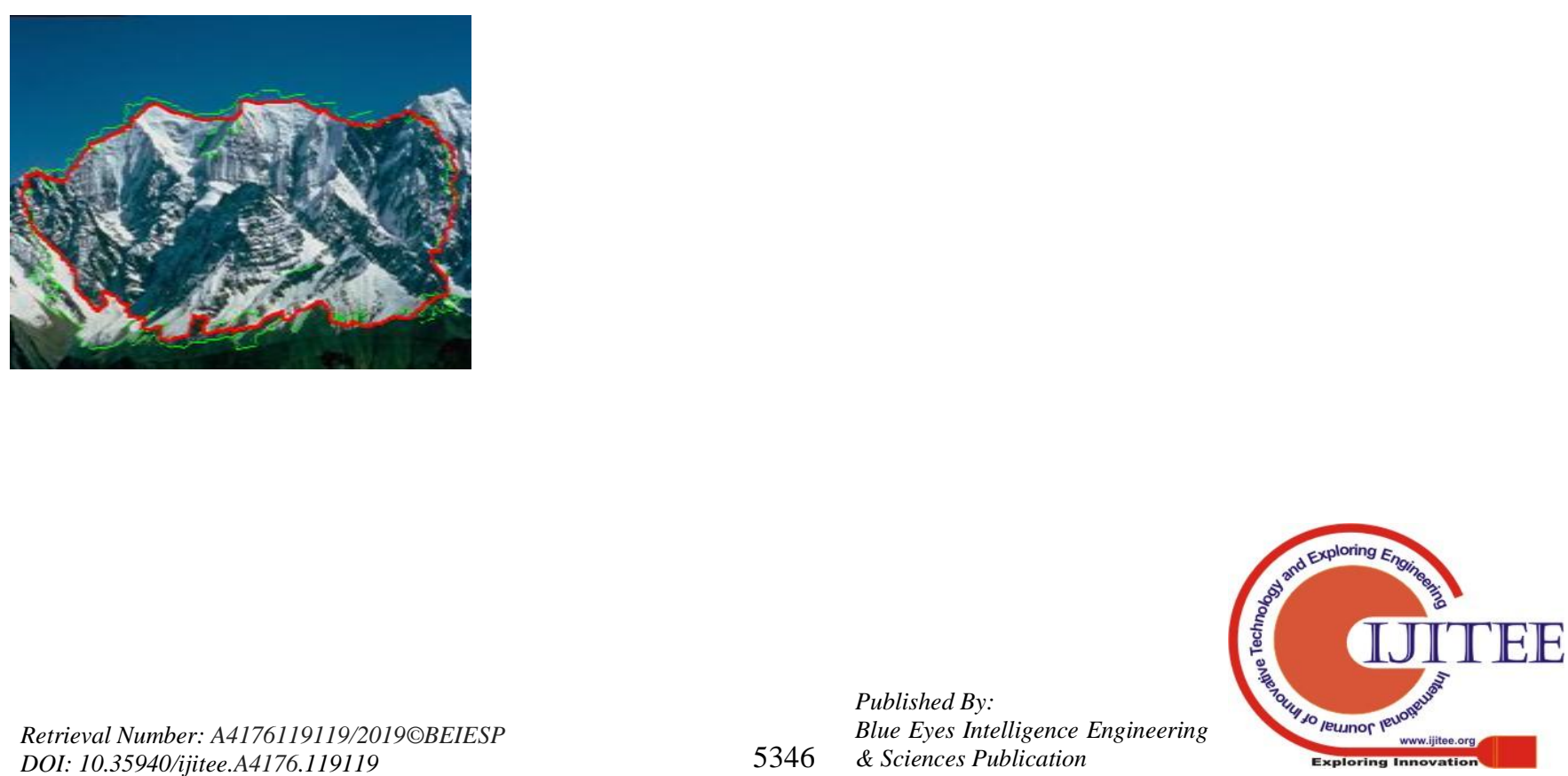


\section{Content Based Image Retrieval based on Domain Knowledge Acquisition}

Figure 19 Query Image of Mountain

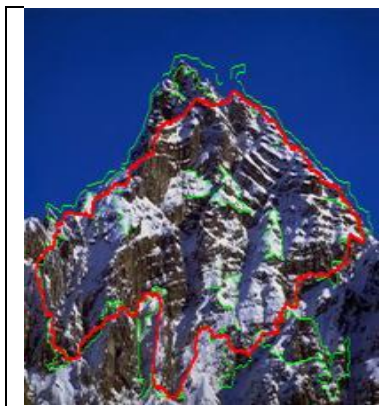

a)

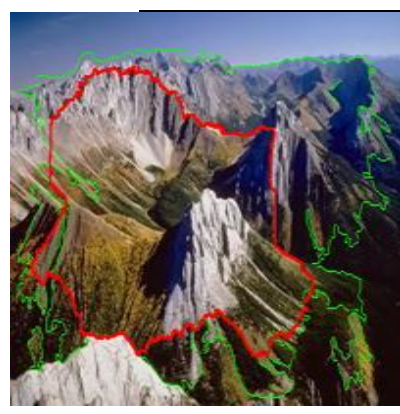

b)

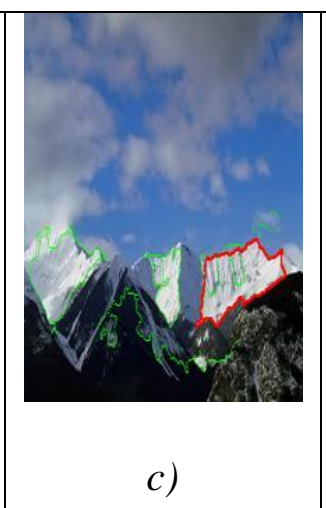

c)

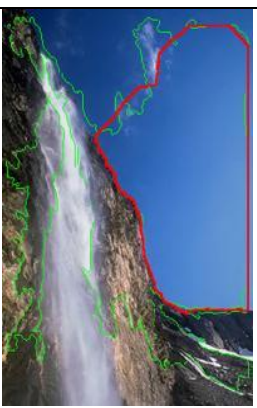

d)

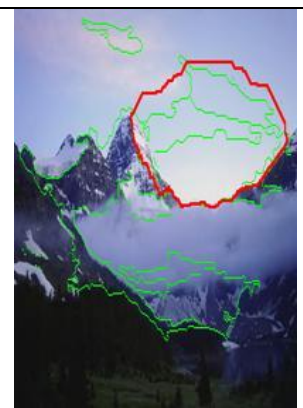

e)

Figure 20 Response Image of Mountain Category

Query Image: 10

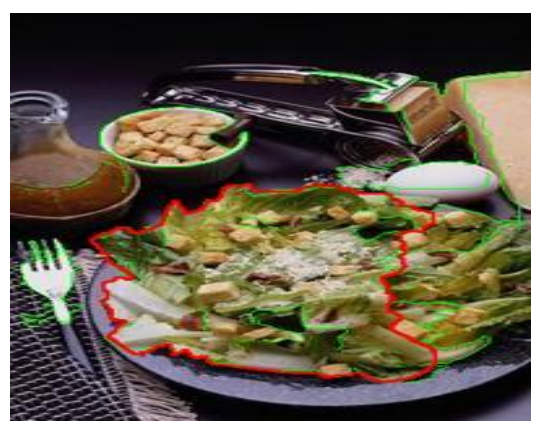

Figure 21 Query Image of Food

Figure 22 Response image of Food Category



a)

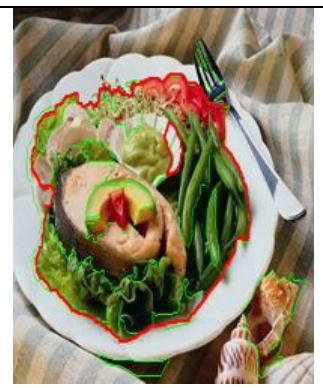

b)

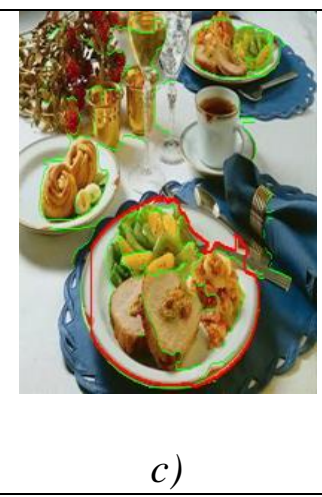

c)
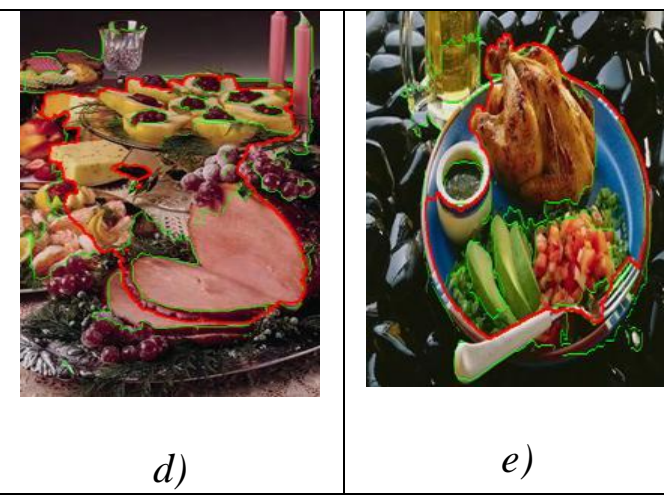

e)

\section{VI.}

Over the last few years, CBIR has drawn the fine attention in the research area to retrieve the image. In this paper, we have presented a novel methodology named as Domain Knowledge Acquisition, which helps in achieving the new similarity measure. Moreover DKA methodology not only helps in achieving the similarity matching in domain but cross domain model. In order to evaluate the DKA methodology extensive simulation was done by using the Corel1k Dataset. In evaluation first we have retrieved the top 5 response of the given query image, furthermore comparative analysis is done with several state-of- art technique and existing method CPV-THF. Moreover precision is considered as the comparing parameter and we observe that average precision value of existing method is less than the proposed. 


\section{REFERENCE}

1. S. Li, S. Chen, S. Su, D. Duh and S. Li, "Graphics/Image Retrieval Method," 2011 International Conference on Technologies and Applications of Artificial Intelligence, Chung-Li, 2011, pp. 19-24.

2. P. Wilkins, P. Ferguson, A. F. Smeaton and C. Gurrin, "Text based collections," The 2nd European Workshop on the Integration of Knowledge, Semantics and Digital Media Technology, 2005. EWIMT 2005. (Ref. No. 2005/11099), London, 2005, pp. 281-288.

3. Long F., Zhang H., Feng D.D. (2003) Fundamentals of ContentBased Image Retrieval. In: Feng D.D., Siu WC., Zhang HJ. (eds) Multimedia Information Retrieval and Management. Signals and Communication Technology. Springer, Berlin, Heidelberg.

4. G. Schaefer, "An introduction to content-based image retrieval," Eighth International Conference on Digital Information Management (ICDIM 2013), Islamabad, 2013, pp. 4-6.

5. P. S. Hiremath and J. Pujari, "Content Based Image Retrieval Using Color, Texture and Shape Features," 15th International Conference on Advanced Computing and Communications (ADCOM 2007), Guwahati, Assam, 2007, pp. 780-784.

6. Lingaraj, Haldurai. (2015). Parallel Indexing on Color and Texture Feature Extraction using R-Tree for Content Based Image Retrieval. International Journal of Computer Sciences and Engineering. 3. 1115.

7. Joseph, T., Cardenas, A. F., "PICQUERY: A High Level Query Language for Pictorial Database Management”, May 1988, vol. 14 no. 5 , pp. $630-638$ selection for texture segmentation", IEEE Transactions, Ju 1989, Volume : 11 Issue : 7, 717 to 728

9. M. Mohammadpour and S. Mozaffari, "A method for Content-Based Image Retrieval using visual attention model," 2015 7th Conference on Information and Knowledge Technology (IKT), Urmia, 2015, pp. $1-5$.

10. Figueiredo, M.A.T.,Hong-Jiang Zhang, Jain, A.K., Vailaya, A., "Image classification for content-based indexing ",IEEE Transactions, 2001

11. Wei Song, Yubing Zhang, Fei Liu, Zhilei Chai, Feng Ding, Xuezhong Qian, Soon Cheol Park,Taking advantage of multi-regions-based diagonal texture structure descriptor for image retrieval,Expert Systems with Applications,Volume 96,2018,Pages 347-357,ISSN 0957-4174.

12. Ghebreab, S., Jaffe, C.C., Smeulders, A.W.M., "Population-based incremental interactive concept learning for image retrieval by stochastic string segmentations", IEEE Transactions , 2004.

13. S. -. Chen, S. H. Rubin, M. -. Shyu and C. Zhang, "A Dynamic User Concept Pattern Learning Framework for Content-Based Image Retrieval," in IEEE Transactions on Systems, Man, and Cybernetics, Part C (Applications and Reviews), vol. 36, no. 6, pp. 772-783, Nov. 2006.

14. Lin Feng, Jun Wu, Shenglan Liu, Hongwei Zhang,Global Correlation Descriptor: A novel image representation for image retrieval,Journal of Visual Communication and Image Representation,

a. Volume 33,2015,Pages 104-114,ISSN 1047-3203

15. W. Jiang, G. Er, Q. Dai and J. Gu, "Similarity-Based Online Feature Selection in Content- Based Image Retrieval", IEEE Trans. on Image Processing, vol. 15, no. 3, pp. 101-104, March 2006.

16. Mutasem K. Alsmadi, Query-sensitive similarity measure for contentbased image retrieval using meta-heuristic algorithm,Journal of King Saud University - Computer and Information Sciences, Volume 30 Issue 3,2018,Pages 373-381.

17. Basak, J., Bhattacharya, K., Chaudhury, S., "Multiple Exemplar-Based Facial Image Retrieval Using Independent Component Analysis", IEEE Transactions, Dec 2006.

18. Chai J. Y., C. Zhang, R. Jin, "An Empirical Investigation of User Term Feedback in Textbased Targeted Image Search", ACM Transaction, Inform. Systems, Vol. 25, 2007, No 1.

19. Ye, L., Zang, J., "Content Based Image Retrieval Using Unclean Positive Examples", Image

a. Processing, IEEE Transactions, Oct 2009

20. Davis, L.S., Zhe Lin, “ Document Title : Shape-Based Human Detection and Segmentation via Hierarchical Part-Template Matching", Pattern Analysis and Machine Intelligence, IEEE Transactions, Apr 2010.

21. Abbadeni, N., "Computational Perceptual Features for Texture Representation and Retrieval", IEEE Transactions, Jan 2011. approaches for content-based image retrieval on large image

8. Eden, M., Unser, M., "Multiresolution feature extraction and

22. Dacheng Tao_3;Dongquan Liu_2;Hock Soon Seah_4;Jun Yu, “ On Combining Multiple Features for Cartoon Character Retrieval and Clip Synthesis", IEEE Transactions, Oct 2012

23. G.-H. Liu, L. Zhang, Y.-K. Hou, Z.-Y. Li, and J.-Y. Yang, "Image retrieval based on multi-texton histogram," Pattern Recognit., vol. 43 pp. 2380-2389, Jul. 2010.

24. G.-H. Liu, Z.-Y. Li, L. Zhang, and Y. Xu, "Image retrieval based on microstructure descriptor," Pattern Recognit., vol. 44, no. 9, pp. 2123 2133,2011.

25. G.-H. Liu and J.-Y. Yang, "Content-based image retrieval using color difference histogram," Pattern Recognit., vol. 46, pp. 188-198, Jan. 2013.

26. X. Wang and Z. Wang, "A novel method for image retrieval based on structure elements' descriptor," J. Vis. Commun. Image Represent. vol. 24, pp. 63-74, Jan. 2013

27. L. Feng, J. Wu, S. Liu, and H. Zhang, "Global correlation descriptor:A novel image representation for image retrieval," J. Vis Commun. Image Represent., vol. 33, pp. 104-114, Nov. 2015.

28. M. Subrahmanyam, Q. M. J. Wu, R. P. Maheshwari, and R. Balasubramanian, "Modified color motif co-occurrence matrix for image indexing and retrieval," Comput. Elect. Eng., vol. 39, no. 3,pp. 762-774, 2013.

29. A. Raza, H. Dawood, H. Dawood, S. Shabbir, R. Mehboob and A. Banjar, "Correlated Primary Visual Texton Histogram Features for Content Base Image Retrieval," in IEEE Access, vol. 6, pp. 4659546616,2018

\section{AUTHORS PROFILE}

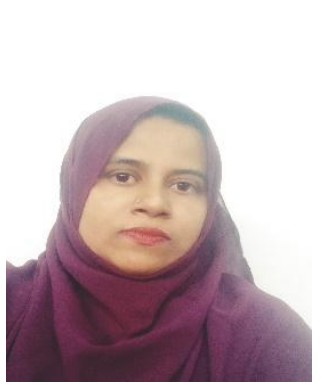

\section{Author-1}

Prof. Feroza M. Mirajkar is working as Assistant Professor in Department of E\&CE, in Khaja Banda Nawaz College of Engineering, Kalaburgi, Karnataka, India since 2013. Completed Master of Technology from Basavesvara College of Engineeringg, Bagalkot, Karnataka, India. She has attended many workshops such as "Innovative research techniques" a National workshop, CUK, Kalaburagi, participitated in a Two-week ISTE STTP on "Pedagogy for Effective use of ICT in Engineering Education" conducted by Indian institute of Institute of Technology Bombay at NK Orchid college of Engineering and Technology, Solapur and many more. She has 12 publications which include both National and international journal and conferences with one IEEE.



\section{Author-2}

Dr. Ruksar Fatima is presently working as Dean FACULTY OF Engineering and Technology, Khaja Bandanawaz University, Gulbarga with an experience of 18 years as a dedicated, resourceful education professional. She has received 4 awards, Award for Best Scientific publication by VGST in 2018, RURLA award for Distinguished Scientist in 2018, Chairman for IETE Gulbarga sub-center and Best Senior Researcher (Female) by international Academic and Research Excellence Awards 2019. She has 31 International publications in reputed journals and technical member and reviewer of many famous international Journals. She has written a book entitled "Image Processing Techniques for classification of Images" https://www.interlinepublishing.com/title cover/large/caf591ac8e3ef54ba4d4046650e5971e.jpg. 


\title{
Content Based Image Retrieval based on Domain Knowledge Acquisition
}

\begin{abstract}
Author-3

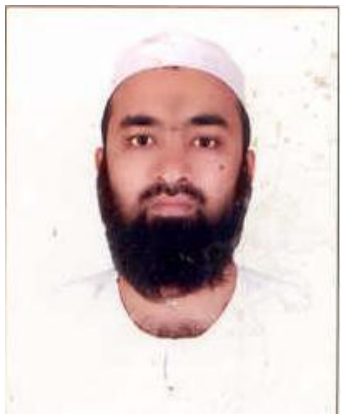

Dr. Shaik A Qadeer is working as a Professor in Muffakham Jah College of Engineering and Technology, Hyderabad, completed M.Tech followed by Ph.D. from reputed University (Jawaharlal Nehru Technological University Hyderabad-Electronics and Communication Engineering). Published 9 international Journals and 9 international conferences, most of the research work is executed with the association of prime universities like Indian Institute of Technology Hyderabad and International Institute of Information Technology, Hyderabad. The publications include foreign conferences conducted in countries the USA, Denmark, and Turkey. Out of 18 publications 6 publications are there in IEEE explorer, eight sponsored projects are successfully completed which is funded by Muffakham Jah College of Engineering and Technology. An Indian patent is filed and published by OFFICIAL JOURNAL OF THE PATENT OFFICE on 17-8 2018 with number 201741035875. Two Book chapters are published in Springer series-Lecture notes in electrical Engineering. He has Achieved 5th rank in University in Bachelor Degree course. The following Certifications are completed .1) "High impact presentation skills" from Dale Carnegie Training 2) "Bringing innovation in teaching and learning process" from Wipro missionX10.
\end{abstract}

